\title{
Prominent Photospheric Downflows on Magnetic Neutral Line in a Delta-Type Sunspot
}

\section{$\operatorname{AUTHOR}(\mathrm{S})$ :}

Takizawa, K.; Kitai, R.; Zhang, Y.

\section{CITATION:}

Takizawa, K....[et al]. Prominent Photospheric Downflows on Magnetic Neutral Line in a Delta-Type Sunspot. Solar Physics 2012, 281(2): 599609

\section{ISSUE DATE:}

2012-12

URL:

http://hdl.handle.net/2433/163490

\section{RIGHT:}

The final publication is available at www.springerlink.com; This is not the published version. Please cite only the published version.; この論文 は出版社版でありません。引用の際には出版社版をご確認ご利用くだ さい。 


\title{
Prominent Photospheric Downflows on Magnetic Neutral Line in a Delta-Type Sunspot
}

\author{
K. Takizawa ${ }^{1} \cdot$ R. Kitai ${ }^{1} \cdot$ Y. Zhang $^{1,2}$ \\ (C) Springer $\bullet \bullet \bullet \bullet$
}

\begin{abstract}
$\delta$ type sunspot groups are known to have high flare productivity and to produce very strong flares. In particular, $\beta \gamma \delta$ type sunspots are the most active type of all. A $\beta \gamma \delta$ active region NOAA 9957 with frequent flux cancelations but without any marked flare activity during its decay phase was studied in this work. Using SOHO/MDI Dopplergrams and magnetograms, we detected continuous prominent downflow motions of $1500-1700 \mathrm{~m} \mathrm{~s}^{-1}$ for several hours on the magnetic neutral line in this region. In the downflow region, penumbral structure were observed to decay. We will interpret and discuss the phenomenon as one case of submergence of the magnetic flux.
\end{abstract}

Keywords: Active Regions, Magnetic Fields, Photosphere

\section{Introduction}

Active regions (ARs) are known to be born as emerging flux regions (EFRs), develop their complexities and then decay after exhibiting explosive activities. Among them, $\beta \gamma \delta$ active regions are known to show highly energetic flare activities (Sammis et al., 2000). However, some $\delta$ regions do not show so much violent activities (Zirin and Liggett, 1987). During the surveying study of $\beta \gamma \delta$ regions, we found an indication of submergence of magnetic flux in an inactive and decaying $\beta \gamma \delta$ region.

We will report the temporal behavior of the region as a case study of magnetic flux decay of the region. In general, the reduction of the observed surface magnetic flux can be attributed to processes such as submergence, rapid or slow dissipation, diffusion by turbulent flow, and reconnection. Submergence was studied by many researchers until now (Wallenhorst and Howard, 1982; Wallenhorst and Topka, 1982; Rabin et al., 1984; Zirin, 1985; Harvey et al., 1999; Kálmán 2001; Chae et al., 2004; Kubo et al., 2010; Iida et al., 2010). Zirin (1985)

\footnotetext{
${ }^{1}$ Kwasan and Hida Observatories, Kyoto University, Yamashina-ku, Kyoto 607-8417, Japan email: takizawa@kwasan.kyoto-u.ac.jp email:

kitai@kwasan.kyoto-u.ac.jp

2 National Astronomical Observatory, Chinese Academy of

Sciences, Beijing 100012, China email: zhangyin@bao.ac.cn
} 


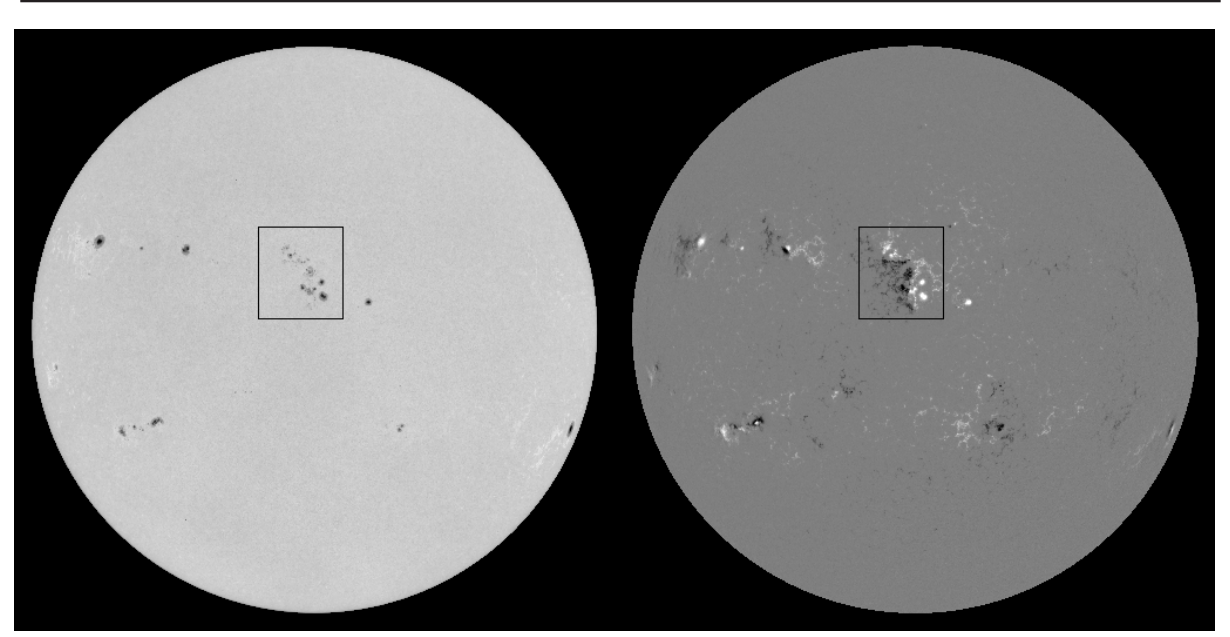

Figure 1. White light image and magnetogram of active region NOAA 9957 observed with SOHO/MDI on 22 May 2002, 11:12 UT.

studied submergence of small dipole in AR BBSO 18962 and proposed that the flux loop was pulled back down by magnetic tension and submerged. Harvey et al. (1999) studied the disappearance timing of magnetic bipoles in different layers (photosphere, chromosphere and corona). They found that the bipoles disappear earlier in the chromosphere and corona than in the photosphere for $44 \%$ of canceling bipoles. They considered that these cases of magnetic flux cancelation are due to submergence. Chae et al. (2004) reported two cases of $1 \mathrm{~km} \mathrm{~s}^{-1}$ downflow near the magnetic neutral line (NL) at small cancelation sites in NOAA 10043, and they considered that the flow corresponds to the submergence of an $\Omega$ shaped magnetic flux tube. Recently, using Hinode/SOT data, Kubo et al. (2010) and Iida et al. (2010) reported respectively small magnetic flux submergence at flux cancelation sites in the quiet region. These previous works reveal that magnetic flux submergence may occur in some flux cancelation sites without any eruption events and that the submergence is a rather common phenomenon in spite of prevailing buoyant character of flux tubes in the solar atmosphere.

Active region NOAA 9957 on which we report in this paper was classified as $\beta \gamma \delta$ type and showed low activities. We studied the region using data from Michelson Doppler Imager (MDI; Scherrer et al., 1995) onboard the Solar and Heliospheric Observatory (SOHO) satellite for photospheric velocity field and we discovered prominent downflow of $1.5 \mathrm{~km} \mathrm{~s}^{-1}$ which lasted for about $17 \mathrm{~h}$ near the NL. The observational result strongly suggests the occurrence of submergence in the decay phase of $\beta \gamma \delta$ regions.

\section{Data and Analysis}

Active region NOAA 9957 was observed for 13 days from 16 May to 28 May 2002 on the solar disk $\left(\mathrm{N}^{\circ}-\mathrm{N} 15^{\circ}\right)$. No flare activities were reported from 20 

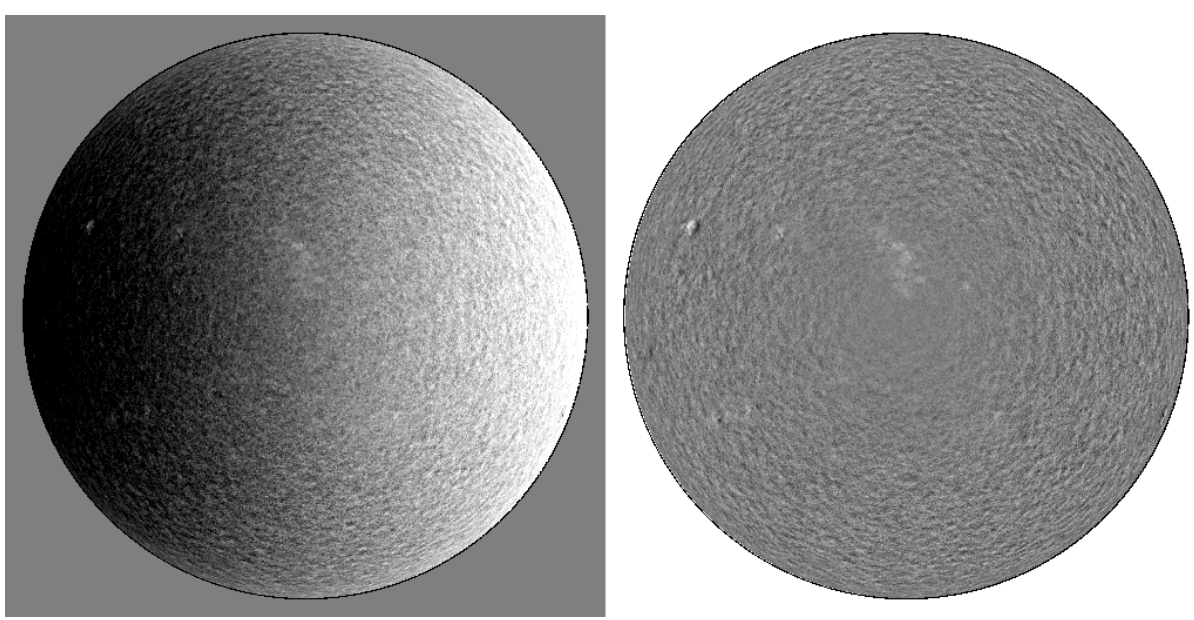

Figure 2. An original MDI Dopplergram (left) and the data after correction for solar rotation and 5 min oscillation (right).

to 26 May in the region, although NOAA 9957 was classified as a $\beta \gamma \delta$ sunspot group during 10 days from 17 to 26 May (Figure 1 ).

In this study, we use MDI Dopplergrams, magnetograms, and continuum images onboard the $\mathrm{SOHO}$ satellite. The data on the magnetic field and white light images have a cadence of $96 \mathrm{~min}$, while Dopplergrams and line of sight (LOS) velocities have a cadence of $1 \mathrm{~min}$. These data have a spatial resolution of 2 arcsec per pixel. Partially, Dopplergrams of 0.6 arcsec per pixel are included for analysis after 20:00 UT, 22 May. Although we describe the analysis method of Dopplergrams taken under the normal mode in the following, we have treated high resolution mode data in a similar manner.

For preparation of our analysis using Dopplergrams, first we removed the effect of solar rotation with the following method. We made a mean Doppler image by averaging all the Doppler images (7689 images) during almost 4 days (from 18 to 22 May), and subtracted the mean image from each image. Secondly, we corrected for the $5 \mathrm{~min}$ oscillation by averaging every sequential four images (Figure 2).

From the time series of magnetograms, we derived the horizontal velocities of magnetic features by the local correlation tracking method (LCT; November et al., 1988) in $96 \mathrm{~min}$ temporal step with a window size of $5 \times 5$ arcsec.

In addition, we applied a labeling method (connected component labeling) to identify notable redshift patches. Our labeling method on binary images is similar to the one described in Gonzalez and Woods (1992). The redshift patches from Dopplergram data were extracted with a criterion that LOS velocities were above $800 \mathrm{~m} \mathrm{~s}^{-1}$. We identified each notable identical patch between consecutive images visually, and investigated their evolution.

We defined the sunspot regions, including penumbrae, as darker ones below 0.9 times the quiet region brightness and measured their area variations. ( $c f$. Brandt et al., 1990; Chapman et al., 1994). 


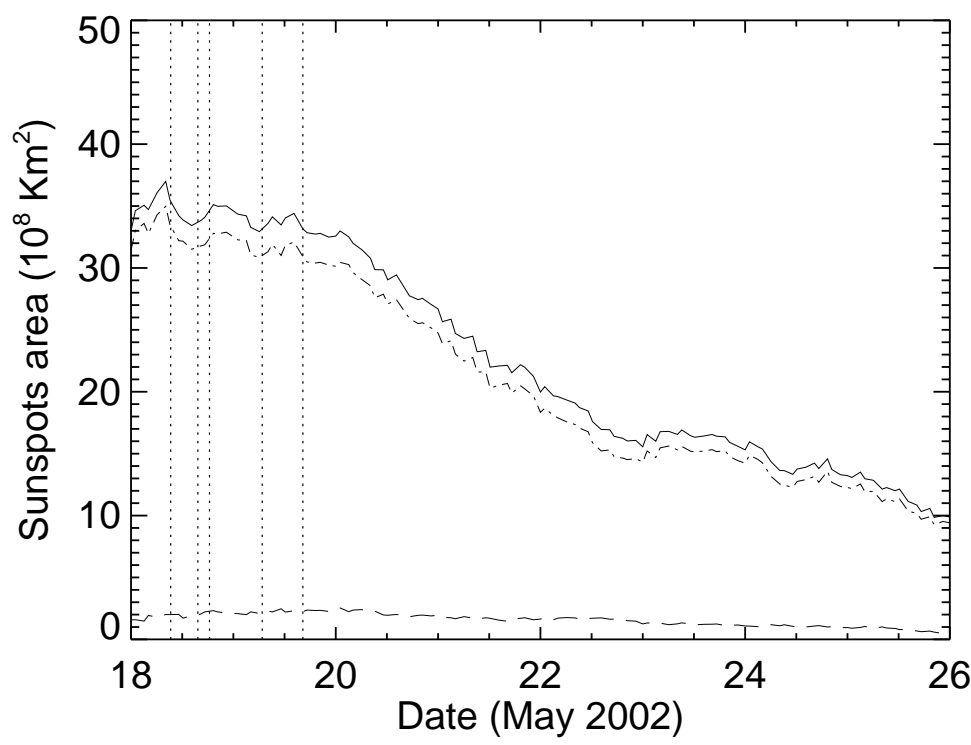

Figure 3. Temporal variation of sunspot area in region NOAA 9957. Solid, dash-dotted, and dashed lines represent sunspot area, penumbral area, and umbral area, respectively. Vertical dotted lines represent the occurrence times of $\mathrm{C}$ class flares by GOES X-ray data.

Magnetic flux values of the region were corrected for the projection effect by dividing each pixel data by $\cos \theta \cos \phi$, where $\theta$ and $\phi$ represent heliographic longitude and latitude, respectively.

\section{Results}

Although NOAA 9957 is the most complex sunspot type, i.e., $\beta \gamma \delta$ region, the area rapidly decreased in the observation period from 20 to 26 May without any flare activity (Figure 3). The region was in the decay phase. We have found several redshift patches around the magnetic neutral line in this region (Figure 4).

\subsection{Case Study of Most Prominent Redshift Patch}

The most prominent downflow (RS6 in Figure 4) appeared in NOAA 9957 from 20 to 21 May 2002 and lasted for about $17 \mathrm{~h}$ at a level of $1.0 \mathrm{~km} \mathrm{~s}^{-1}$. RS6 was located at $5^{\circ}$ north in latitude. As can be seen in figure 5 , the velocity contour of $300 \mathrm{~m} \mathrm{~s}^{-1}$ includes both magnetic polarity areas. RS6 disappeared suddenly and penumbral structures in RS6 decayed simultaneously. Visual inspection of magnetogram movies tells us that moat flows continued steadily from the two stable sunspots of positive polarity located west of the NL during the observation period. Neighboring negative polarities also moved on from the east side to the 

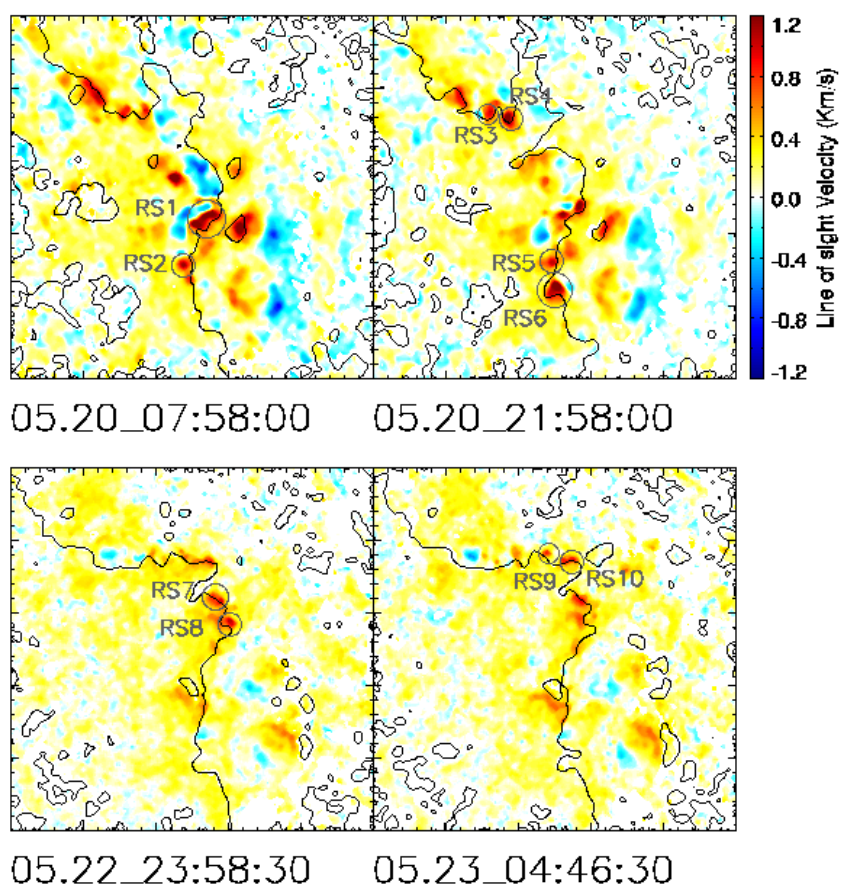

Figure 4. Temporal evolution of Doppler velocities in active region NOAA 9957. The field of view (FOV) is $250^{\prime \prime} \times 250^{\prime \prime}$ for each panel. Magnetic neutral lines are overlaid with black lines. Prominent redshift patches are indicated with circles. North is up and west to the right in these and subsequent maps.

NL. These converging horizontal flows to the NL in the downflow area were also confirmed by the LCT method. Figure 6 shows that the converging flow continued from around 00:00 UT to 04:47 UT on 21 May for about $5 \mathrm{~h}$ when the downflow area (RS6) was growing. The converging flows weakened after the decline of the downflow.

Figure 7 shows the temporal variation of LOS velocity for the prominent redshift patch (RS6). For comparison, the time variation of LOS velocity associated with the Evershed flow (its redshift part) is also shown. In this figure, the abscissa is the distance from the disk center. The representative median values of LOS velocity in these areas are found to be above $500 \mathrm{~m} \mathrm{~s}^{-1}$. The redshifted flow in RS6 showed no pairing blueshifted area; it showed transient behavior, different evolution, and larger Doppler velocity compared to the Evershed flow. Therefore, the flow in RS6 is not due to the Evershed flow. Furthermore, if the observed redshift is due to horizontal flows, the dependence of Doppler amplitude on the distance from the disk center will be similar to the Evershed flow. As the Doppler shift in RS6 behaves so differently, we may conclude that the observed Doppler shift is mainly due to downflow to lower layers.

Next let us consider the magnetic field around RS6. The vector magnetogram obtained at Huairou Solar Observatory Station, National Astronomical Obser- 

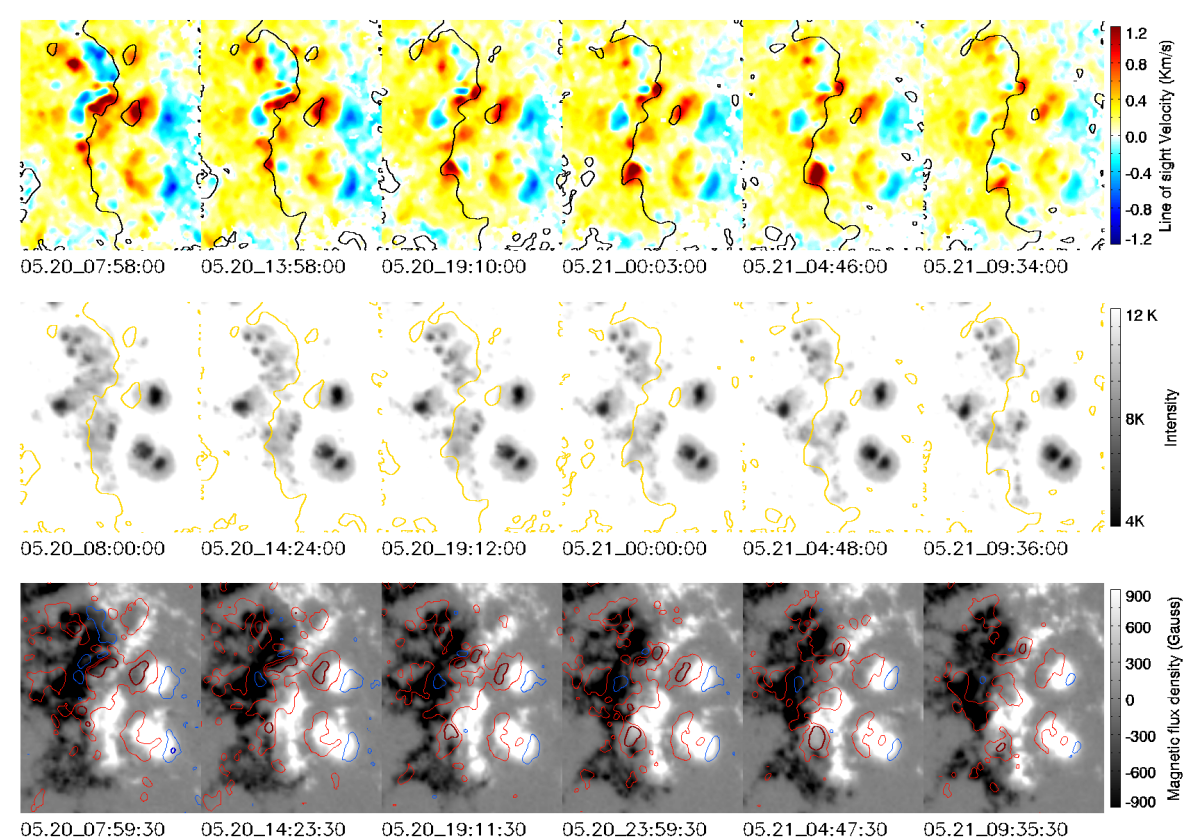

Figure 5. Temporal evolution of the most prominent downflow (RS6). The FOV is $130^{\prime \prime} \times$ $165^{\prime \prime}$ for each panel. Time series of Dopplergrams, white light images, and magnetograms are shown from left to right for each row. Magnetic neutral lines are drawn in the panels at the top and middle rows. In the panels at the bottom row, dark red, light red, and light blue contours represent $800 \mathrm{~m} \mathrm{~s}^{-1}$ redshift, $300 \mathrm{~m} \mathrm{~s}^{-1}$ redshift, and $300 \mathrm{~m} \mathrm{~s}^{-1}$ blueshift, respectively. The dark blue contour which represents $800 \mathrm{~m} \mathrm{~s}^{-1}$ blueshift is only in the bottom far left panel on the positive polarity sunspot at south-west.

vatories of China (Figure 8) shows that magnetic lines of force ran parallel to the solar surface in the RS6 area. Continuous downflow acting on horizontal magnetic fields would lead to decay of penumbral structures. These observed results strongly suggest the occurrence of a submergence of magnetic $\Omega$ loop structure in the area.

\subsection{Statistical Study of Redshift Patches}

We investigated all the redshift patches in NOAA 9957 that showed redshift above $800 \mathrm{~m} \mathrm{~s}^{-1}$. The following criteria were used for selection of the patches: They (1) had redshift above $800 \mathrm{~m} \mathrm{~s}^{-1}$, (2) occurred near the NL, (3) were located within about $30^{\circ}$ of the central meridian, (4) had no characteristics of the Evershed flow, (5) were clearly identifiable over a period longer than $4.5 \mathrm{~h}$ (to be found at least four consecutive MDI magnetograms with 96 min cadence).

As a result, we found nine cases which satisfied the criteria above. We studied the correlation between their LOS velocities and life times and summarized the result in Figure 9. The maximum velocity during the life time of each patch is taken as a representative value. We can see that almost all of redshift patches 


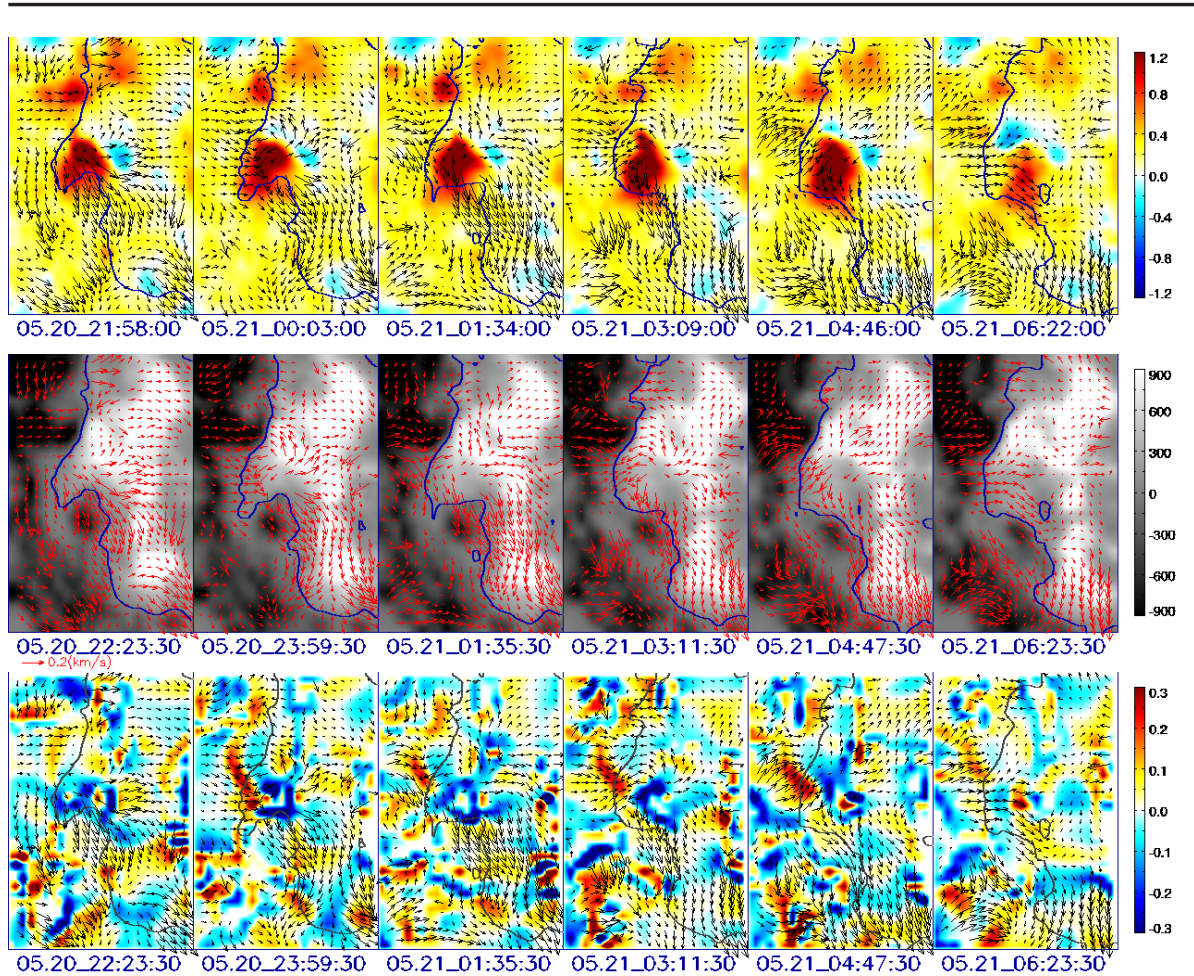

Figure 6. Temporal evolution of horizontal flow. The FOV is $50^{\prime \prime} \times 75^{\prime \prime}$ for each panel. LCT flow vectors are shown by arrows on the Doppler maps at the top row and on the magnetic maps at the middle row, respectively. Panels at the bottom row show divergence of horizontal velocities; reddish colors indicate convergence and bluish colors indicate divergence.

have velocities around $1 \mathrm{~km} \mathrm{~s}^{-1}$ at maximum, and these values do not depend on their life time. Majority of them have life time less than $20 \mathrm{~h}$ and they show pulselike temporal evolution as that of RS6. Their transient activities were observed to be intermittent.

\section{Discussion and Conclusions}

There are several possibilities for disappearance of active regions: magnetic diffusion, magnetic reconnection, submergence, etc. Active region NOAA 9957 in this study reduced its penumbral area $46 \%$ from 20 May 00:00 UT to 22 May 12:00 UT. The rate of decrease of the penumbral area was $22 \%$ per day over the period of 2.5 days.

Our study shows that the decay in penumbral structures mainly occurred in the vicinity of the NL, and must be strongly associated with magnetic flux cancelations. For instance, in a very small area $\left(20^{\prime \prime} \times 25^{\prime \prime}\right)$ including RS6, the magnetic flux dropped by $30 \%$ during only one day (Figure 10), and this reduction was accompanied by a dramatic decay in the penumbral structures 


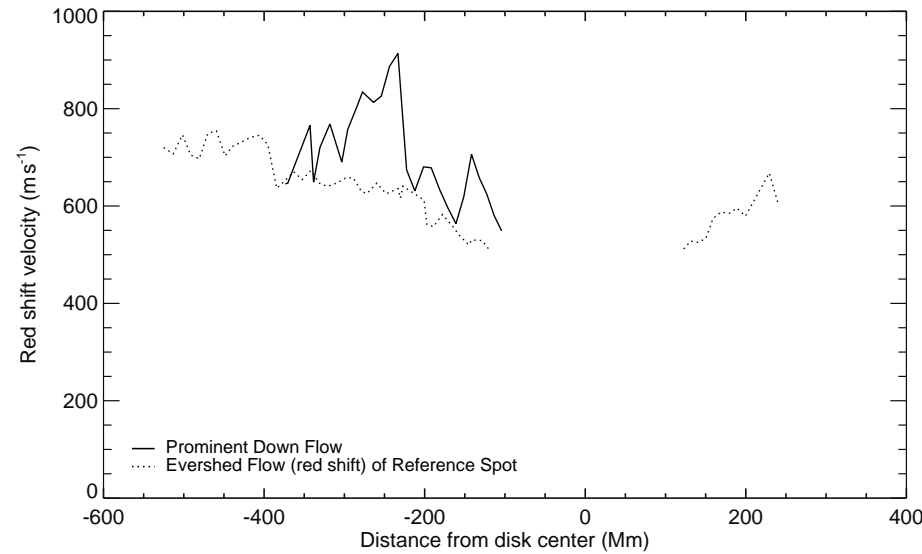

Figure 7. The solid line represents the temporal evolution of prominent downflow in RS6. For comparison, the dotted line represents the time evolution of the redshift portion of the Evershed flow in the reference round sunspot NOAA 9958. The velocities are median values in the area surrounded by the contour of $500 \mathrm{~m} \mathrm{~s}^{-1}$ LOS velocity.

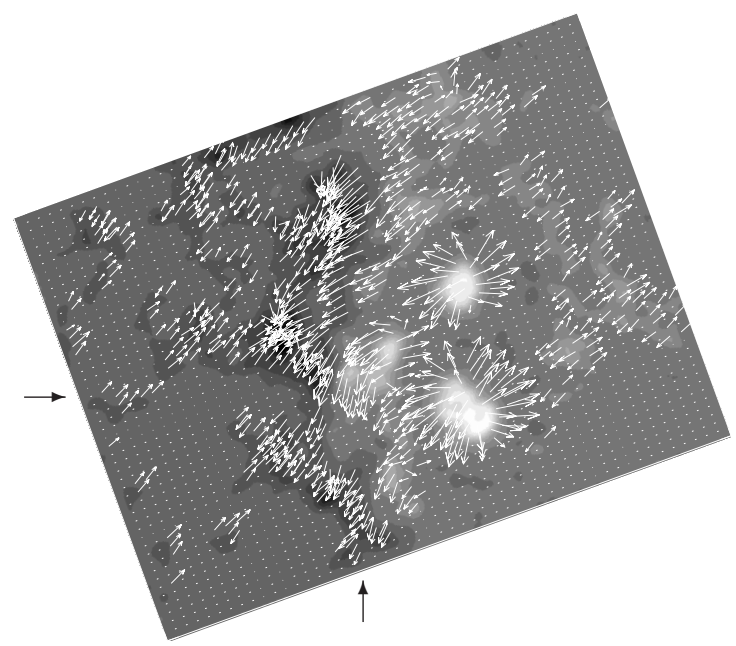

Figure 8. Vector magnetogram obtained at Huairou Solar Observatory Station of NAOC, 21 May 2002, 0350 UT. RS6 is at the intersection of outer two black arrows. Magnetic lines of force ran parallel to the solar surface in the RS6 area.

that existed in RS6. We consider that the downflow activities may have caused rapid decay of this AR. In the following we will discuss possible mechanisms of the rapid decay of NOAA 9957.

First, let us consider the diffusion mechanism. Howard and LaBonte (1981) examined magnetic field measurements carried out at Mt. Wilson from 1967 to mid-1980. They found that the rate of disappearance of magnetic flux from 


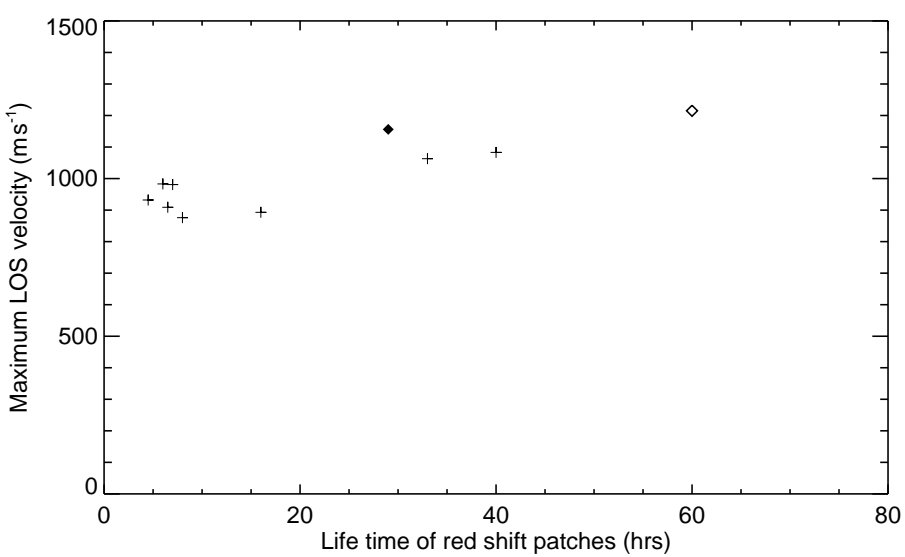

Figure 9. Maximum LOS velocities during the life time of various redshift patches near the NL in NOAA 9957. The velocities shown are median values computed in the area surrounded by the contour of $800 \mathrm{~ms}^{-1}$ LOS velocity. The filled diamond represents RS6 which is described in Section 3.1 as a case study. The empty diamond represents RS1 and is shown only for reference: $\mathrm{RS} 1$ was observed far from the central meridian $\left(\approx 45^{\circ}\right.$ east in longitude) and may be influenced by horizontal flow.

active latitudes is about $10 \%$ per day of the total flux. They also pointed out that the disappearance of active region fields is not due to diffusion, but advection to polar latitudes by meridional flow. Wallenhorst and Howard (1982) checked temporal evolution of average magnetic flux for 25 ARs. Their analysis indicated that the average rate of flux disappearance is also about $10 \%$ per day. The weak background fields below $10 \mathrm{G}$ (gauss) around ARs show no increase compensating for the decrease of total magnetic flux above $10 \mathrm{G}$. Thus, they concluded that diffusion does not play an important role in the disappearance of the flux. Wallenhorst and Topka (1982) also reported similar results for a small sunspot group associated with Hale AR 17694.

Second, the possibility of reconnection would be checked. When magnetic reconnection occurs frequently in these events, we can expect that the magnetic flux will decrease rapidly and that X-ray emission will be enhanced. As GOES $\mathrm{X}$-ray intensity was at low level and did not show strong flares during our period of study, we can rule out the reconnection as the mechanism of the decay of NOAA 9957.

Finally, possibility of submergence will be considered. Let us discuss the directions of flows in the redshift patches. Are they horizontal or vertical? As mentioned above in Section 3.2, the strong Doppler signals observed near the NL are almost all redshifts. If we suppose this phenomenon as due to horizontal flows seen from a certain direction, the Doppler signals must change from red to blue (or from blue to red) when the patches cross the central meridian. However, we never detected such an effect. When the horizontal flows have random directions, it is highly likely that both redshift and blueshift will be found. Therefore, this 

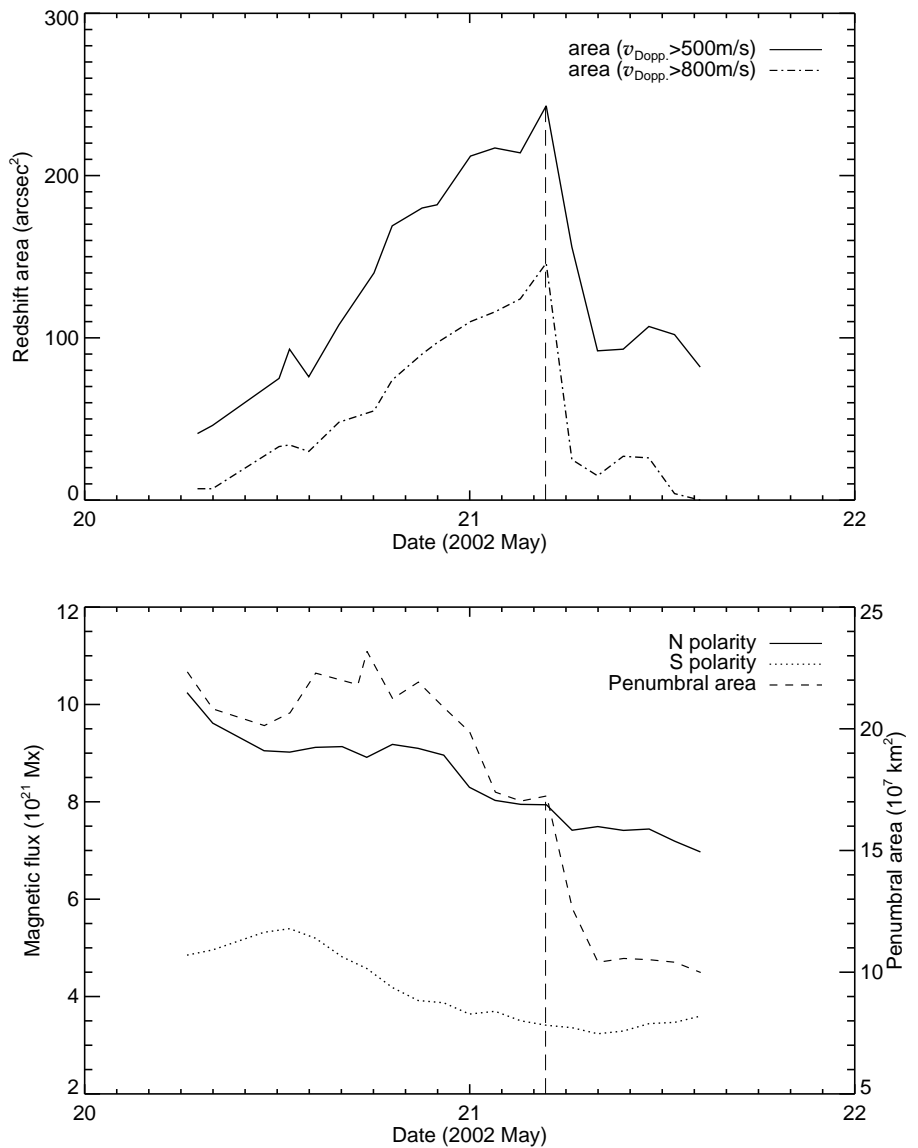

Figure 10. Top panel shows area changes in RS6. The solid and dash-dotted lines correspond to the area defined by Doppler velocities exceeding $500 \mathrm{~ms}^{-1}$ and $800 \mathrm{~ms}^{-1}$, respectively. Bottom panel shows temporal evolutions of the magnetic flux and penumbral area in RS6. The solid, dotted, and dashed lines represent positive polarity flux, negative polarity flux, and penumbral area, respectively. The vertical dashed lines in both panels represent the time when the penumbral area showed dramatic decay.

possibility is ruled out from the observational results, and the flows in the redshift patches will be vertical.

Another argument supports the view of the vertical flow. RS7 took place near the meridian $\left(\mathrm{W} 6^{\circ}-\mathrm{W}^{\circ}, \mathrm{N} 13^{\circ}\right)$ and always indicated redshift velocity of around $1 \mathrm{~km} \mathrm{~s}^{-1}$. If the redshift is due to horizontal flow, the absolute flow speed reaches $4 \mathrm{~km} \mathrm{~s}^{-1}$. It is not likely that such a phenomenon of sonic-speed level occurs in the photosphere and lives for several hours, which have never been reported.

In case of RS6 as mentioned in Section 3.1, the converging horizontal flows near the NL would enhance the gas density there, and will turn to downward directions around the NL by the gravitational force. We also notice that the converging flows will carry the foot points of magnetic flux tubes to the NL. We 

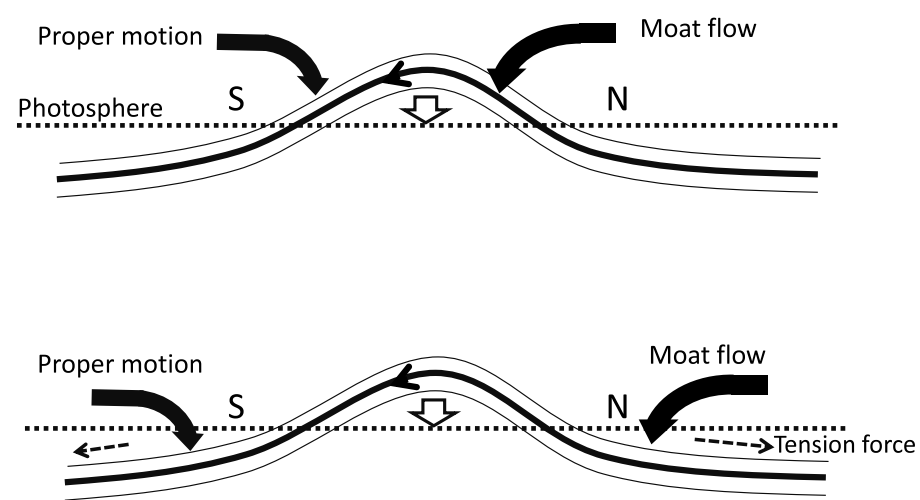

Figure 11. Schematic images of magnetic flux submergence. Top panel illustrates that the moat flows mainly act on the magnetic flux above the solar surface and directly drag the flux downwards. Bottom one shows that the moat flows act on the flux below the surface and enhance the magnetic tension force.

expect that these effects will drive downward motion of magnetic flux tubes near the NL as will be discussed in the next paragraphs.

We propose the following physical pictures for explaining the phenomenon in Figure 11. Moat flows from the two positive polarities adjacent to the strong downflow area continued during the observational period. The proper motion of negative polarities from east to the NL also continued for a long duration $(19-22$ May). There are two possibilities on how the drag force due to the moat flows works on the magnetic flux; either above or below the solar surface. One possibility is that the moat flows mainly act on the magnetic flux above the solar surface and directly drag the flux downwards.

Another possibility is that the moat flows act on the magnetic flux below the surface and enhance the magnetic tension force. Since the magnetic buoyancy is balanced with the magnetic tension force in an emerged and stabilized magnetic flux tube, we obtain the following relation ( $c f$. Parker, 1955, 1984; Priest, 1982):

$$
\left(\rho_{\mathrm{e}}-\rho_{\mathrm{i}}\right) g \approx \frac{B_{\mathrm{i}}^{2}}{\mu L_{\mathrm{c}}},
$$

where $\rho_{\mathrm{e}}$ and $\rho_{\mathrm{i}}$ represent the mass densities outside the tube and inside the tube, respectively, and $L_{\mathrm{c}}$ is the critical separation of the anchor points for an emerging tube. Pariat (2004) pointed out that $L_{\mathrm{c}}$ is around $2000 \mathrm{~km}$. The converging flow as shown in Figure 11 will reduce the separation of the anchor points of a bipole. When $L$ becomes smaller than $L_{\mathrm{c}}$, the magnetic tension force overcomes the magnetic buoyancy and the magnetic bipole will submerge. As the flows from both sides of the NL continued for $5 \mathrm{~h}$ with the velocity of $0.1-0.2 \mathrm{~km} \mathrm{~s}^{-1}$ at the surface as shown in Figure 6, both foot points that were originally separated by $7000 \mathrm{~km}\left(\approx 10^{\prime \prime}\right)$ will come together to $\approx 2000 \mathrm{~km}$. Therefore the foot-point 
separation of the magnetic tubes near the NL become smaller than $L_{\mathrm{c}}$ and the submergence of tubes will be driven near the NL.

This simple estimation tells us that continuous converging flow near the NL can trigger the submergence not only in $\delta$ type sunspots as NOAA 9957 but also in any other types of sunspots. If the magnetic tube lies nearly horizontally and weakly convex to the vertical, submergence is likely to be driven by converging horizontal flows.

During the disk passage of NOAA 9957, there were many redshift patches near the NL as described in Section 3.2. So we can presume that these phenomena of submergence are rather common although they have not been detected masked by other violent activities.

We may summarize our conclusions on these phenomena in the AR NOAA 9957 as follows.

(1) In a rapidly decaying $\beta \gamma \delta$ type AR NOAA 9957, we have detected continuous and prominent downflow patches on the magnetic neutral line.

(2) The downflow motions occurred intermittently with an average lifetime of $12 \mathrm{~h}$.

(3) Near the downflow area, converging horizontal flow to the NL from both sides was found to continue for almost $5 \mathrm{~h}$. We suppose that the moat flows from two large positive polarities promote or drive the phenomenon.

(4) Several submergences occurred along the NL which were accompanied by the flux cancelations and low flare activities.

We suggest that these phenomena may be one of the mechanisms of active region decay irrespective of sunspot types.

Acknowledgements We appreciate very much for fruitful comments by an anonymous referee. This work was supported by the Grant-in-Aid for the Global COE Program "The Next Generation of Physics, Spun from Universality and Emergence" from the Ministry of Education, Culture, Sports, Science and Technology (MEXT) of Japan, and also partly supported by the grant-in-aid from the Japanese MEXT (No. 23540264).

\section{References}

Brandt, P.N., Schmidt, W., Steinegger, M.: 1990, Solar Phys. 129,191.

Chae, J., Moon, Y.-J., Pevtsov, A.A.: 2004, Astrophys. J. 602, 65.

Chapman, G.A., Cookson, A.M., Dobias, J.J.: 1994, Astrophys. J. 432, 403.

Gonzalez, R., Woods, R, :1992, Digital Image Processing, Addison-Wesley, Boston, Chap. 2.

Harvey, K.L., Jones, H.P., Schrijver, C.J., Penn, M.J.: 1999, Solar Phys. 190, 35.

Howard, R., LaBonte, B.J.: 1981, Solar Phys. 74, 131.

Iida, Y., Yokoyama, T., Ichimoto, K.: 2010, Astrophys. J. 713, 325.

Kálmán, B.: 2001, Astron. Astrophys. 371, 731.

Kubo, M., Low, B.C., Lites, B.W.: 2010, Astrophys. J. 712, 1321.

November, L.J., Simon, G.W.: 1988, Astrophys. J. 333, 427.

Pariat, E., Aulanier, G., Schmieder, B., Georgoulis, M. K., Rust, D. M., Bernasconi, P. N.: 2004, Astrophys. J. 614, 1099.

Parker, E.N.: 1955, Astrophys. J. 121, 491.

Parker, E.N.: 1984, Astrophys. J. 280, 423.

Priest, E.R.: 1982, Solar Magneto-hydrodynamics, D. Reidel, Dordrecht, 291.

Rabin, D., Moore, R., Hagyard, M.J.: 1984, Astrophys. J. 287, 404.

Sammis, I., Tang, F., Zirin, H.: 2000, Astrophys. J. 540, 583 
Scherrer, P.H., et al. : 1995, Solar Phys. 162, 129

Wallenhorst, S.G., Howard, R.: 1982, Solar Phys. 76, 203.

Wallenhorst, S.G., Topka, K.P.: 1982, Solar Phys. 81, 33.

Zirin, H.: 1985, Astrophys. J., 291858.

Zirin, H., Liggett, M.A.: 1987, Solar Phys. 113, 267. 
